\title{
Efficiency of Mesoscopic Detectors
}

\author{
S. Pilgram ${ }^{1}$ and M. Büttiker ${ }^{1}$ \\ ${ }^{1}$ Dept. Phys. Théorique, Université de Genève, 24, quai Ernest-Ansermet, 1211 Genève 4, Switzerland
}

\begin{abstract}
We consider a mesoscopic measuring device whose conductance is sensitive to the state of a two-level system. The detector is described with the help of its scattering matrix. Its elements can be used to calculate the relaxation and decoherence time of the system, and determine the characteristic time for a reliable measurement. We derive conditions needed for an efficient ratio of decoherence and measurement time. To illustrate the theory we discuss the distribution function of the efficiency of an ensemble of open chaotic cavities.
\end{abstract}

PACS numbers: 03.65.Ta, 05.45.-a, 73.23.-b, 03.67.Lx

Mesoscopic physics is evolving toward a stage where the understanding of the measurement process becomes of increasing importance. Of interest are detectors which allow a fast determination of the state of the system but at the same time leave the coherence of the measured system as unaffected as possible. These are conflicting requirements: For instance a tunnel contact is an efficient but slow detector. Therefore, the question arises whether it is possible to develop detectors which are both fast and efficient. To answer this question we investigate mesoscopic multichannel conductors and analyze their speed and efficiency. The efficiency of a detector is determined by the ratio of the measurement time and the decoherence time of the measured system.

The effect of a detector on a phase coherent mesoscopic system has been elegantly demonstrated in recent experiments [1] 3]. Theoretical discussions addressed different aspects of weak measurement in mesoscopic systems: the relation to scattering theory [1, 4,5 , and screening [5], the measurement time and interactions [6] and the relation between detector noise and decoherence rate [7] 8]. The time evolution of system and detector has been studied using a master equation approach [9, 10]. Refined calculations consider the conditional evolution of the system depending on the outcome of the measurement [11.12]. Tunnel contacts and single electron transistors have been identified as candidates for efficient measurement devices 13.

Both the measurement time and the decoherence rate depend on the scattering matrix of the conductor (detector). Consequently both of these quantities depend on the sample specific geometry and impurity distribution of the detector. It is therefore necessary to investigate the distribution of the quantities of interest (measurement time, decoherence rate and efficiency) of ensembles of macroscopically identical detectors. Here we focus on ballistic detectors for which ensemble members differ only in their geometry.

The model we consider is shown in Fig. 1. It consists of a double dot (DD) that plays the role of the system. It is an effective two-level system: The topmost electron in the DD can either occupy the upper or the lower dot. The detector is a mesoscopic two-terminal conductor (MC): Its conductance is sensitive to the charge on the upper dot. The coupling between system and detector is de- scribed by a set of capacitances $C_{1}, C_{2}, C_{i}$ that link the charge $Q$ on the $\mathrm{MC}$ to the charges on the dots $Q_{1}$ and $-Q_{1}$ (we abbreviate $C^{-1}=C_{1}^{-1}+C_{2}^{-1}+C_{i}^{-1}$ ).

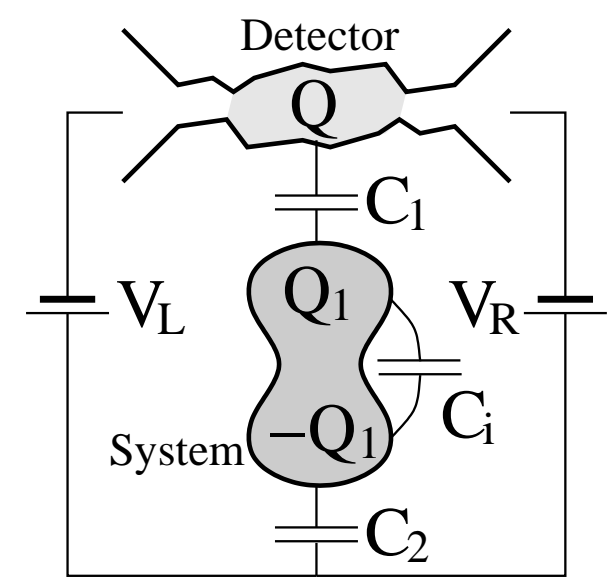

FIG. 1. A mesoscopic detector is capacitively coupled to one side of a double dot.

The interaction of DD and MC can be investigated from two different viewpoints. From the system side we are interested in the question of how fast a pure state prepared in the two-level system decays into a statistical mixture. We distinguish the thermal relaxation to an equilibrium distribution (described by a rate $\Gamma_{r e l}$ ) and the often much faster decoherence of superpositions of states in the upper and lower dot (described by a rate $\left.\Gamma_{d e c}\right)$. This decoherence depends on the temperature $k T$ as well as on the potential difference $e V$ applied at the MC. From the detector side we may ask how long it takes to measure the state of the two-level system (described by a rate $\Gamma_{m}$ ). The decoherence rate at zero temperature $\Gamma_{v}$ is intimately related to the measurement rate $\Gamma_{m}$ and satisfies the inequality $\Gamma_{v} \geq \Gamma_{m}$ 10,14].

We are interested in the conditions under which a MC turns out to be an efficient detector, i.e. fulfills $\Gamma_{v} \gtrsim \Gamma_{m}$. In order to be able to describe a wide class of detectors we represent the MC by a scattering matrix $s_{\alpha \beta}$ that connects in- and outgoing states $(\alpha, \beta$ label left and right reservoir). This enables us to treat multi-channel MCs 
with arbitrary transmission probabilities $T_{n}$ and to include screening effects between different channels. On the other hand a minimum effort is put into the description of the coupling between system and detector. We use a standard master equation (Bloch-Redfield approach [15]) in lowest order perturbation theory to study the evolution of the reduced density matrix of the DD. On this level of approximation the dynamics of the DD is influenced by the charge fluctuation spectrum $S_{Q Q}$ of the MC. A crucial role is therefore attributed to the Wigner-Smith time delay matrix ( $\beta \gamma$ label the reservoirs)

$$
N_{\beta \gamma}=\frac{1}{2 \pi i} \sum_{\alpha} s_{\beta \alpha}^{\dagger} \frac{d s_{\gamma \alpha}}{d E}
$$

that characterizes fully the low-frequency charge fluctuations 16. We introduce the following four constants $(e$ denotes the electron charge)

$$
\begin{gathered}
D=e^{2} \operatorname{Tr} N, \quad C_{\mu}^{-1}=C^{-1}+D^{-1}, \\
R_{q}=\frac{1}{2} \frac{\left(\operatorname{Tr} N^{2}\right)}{(\operatorname{Tr} N)^{2}}, \quad R_{v}=\frac{\left(\operatorname{Tr} N_{12} N_{21}\right)}{(\operatorname{Tr} N)^{2}} .
\end{gathered}
$$

These constants have been applied in many different contexts such as ac-transport and noise $[17,18$. $D$ corresponds to the density of states at Fermi energy in the scattering region, $C_{\mu}$ is an effective electrochemical capacitance that characterizes the strength of interaction, $R_{q}$ expresses the equilibrium contribution to the charge fluctuation spectrum $S_{Q Q}$, and $R_{v}$ the non-equilibrium contribution.

The two-level system is conventionally represented by the Hamiltonian $\hat{H}_{D D}=\frac{\epsilon}{2} \hat{\sigma}_{z}+\frac{\Delta}{2} \hat{\sigma}_{x}$ where $\hat{\sigma}_{i}$ denote Pauli matrices. The energy difference between upper and lower dot is $\epsilon$ and $\Delta$ accounts for tunneling between the dots. The full level splitting is thus $\Omega=\sqrt{\epsilon^{2}+\Delta^{2}}$.

For the relaxation and decoherence rate in the DD we find the following expressions:

$$
\begin{gathered}
\Gamma_{r e l}=2 \pi \frac{\Delta^{2}}{\Omega^{2}}\left(\frac{C_{\mu}}{C_{i}}\right)^{2} R_{q} \frac{\Omega}{2} \operatorname{coth} \frac{\Omega}{2 k T}, \\
\Gamma_{d e c}=2 \pi \frac{\epsilon^{2}}{\Omega^{2}}\left(\frac{C_{\mu}}{C_{i}}\right)^{2}\left(R_{q} k T+R_{v} e|V|\right)+\Gamma_{r e l} / 2 .
\end{gathered}
$$

Eq. (3, (4) are the central result of this paper. It has formally the same appearance as the rates given in [10]. Its big virtue lies in the fact that the structure of the detector is condensed into the four parameters given in (2). An analysis of its properties reduces hence to a discussion of a few parameters. We postpone this discussion and explain first the derivation of Eqs. (3, 4) in order to clarify the approximations made.

The Coulomb energy of our model can be found by circuit analysis

$$
\hat{H}_{C}=\frac{\left(\hat{Q}_{1}-\bar{Q}_{0}\right)^{2}}{2 C_{i}}+\frac{\hat{Q}_{1} \hat{Q}}{C_{i}}+\frac{\hat{Q}^{2}}{2 C} .
$$

Its first term contributes to the level splitting of the DD $\left(\bar{Q}_{0}\right.$ is a background charge depending on the applied voltage $\left.\left(V_{L}+V_{R}\right) / 2\right)$. The charging energy $e^{2} / 2 C_{i}$ must be large compared to $k T, e|V|$ to allow us to consider only two levels of the DD. The second term $\hat{Q}_{1} \hat{Q} / C_{i}$ couples system and detector. In the derivation of the master equation for the reduced density matrix of the system we assume weak coupling and treat this term perturbatively. We apply a Markov approximation which is strictly speaking only valid at long time scales (compared to the correlation time of the detector) and therefore get pure exponential relaxation and decoherence. The third term influences the fluctuation spectrum of the charge operator $\hat{Q}$. In contrast to earlier work (with the exception of Ref. [5]) we do not completely disregard this term, but include it on the level of RPA. This Gaussian approximation restricts us to geometries, where Coulomb blockade effects are weak.

We will now discuss the meaning of the parameters given in Eq. (2) which describe the relation between detector geometry and relaxation or decoherence on the DD.

The parameter $R_{q}$ lies always in the range $1 / 2>R_{q}>$ $1 / 2 N$ where $N$ is the dimension of the scattering matrix. This observation indicates already that the relaxation and decoherence rates $\Gamma_{r e l}, \Gamma_{d e c}$ do not simply scale with the number of channels through the system. It is important to note that the multichannel result for the relaxation and decoherence rates cannot be obtained as a sum of rates due to each channel. For a large number $N$ of open channels $R_{q}$ behaves as $\ln (N) / N$ whereas the electrochemical capacitance $C_{\mu} \rightarrow C$ tends to a constant. The constant $R_{v}$ decreases even stronger than $R_{q}$ like $1 / N$. We find therefore the somewhat surprising result that relaxation and decoherence decrease in the large channel limit. This result is a consequence of screening in the $\mathrm{MC}$ which reduces the charge fluctuations with increasing channel number $N$.

It is interesting to note that the charge response $D \propto$ $\sum \tau_{n}$ can be expressed entirely by the dwell times $\tau_{n}$ which are eigenvalues of the matrix (11). This is also the case for the thermal relaxation parameter $R_{q} \propto$ $\sum \tau_{n}^{2} /\left(\sum \tau_{n}\right)^{2}$. For the thermal charge fluctuations it is unimportant whether a scattering state is connected to the left or the right reservoir. This is reflected by the fact that the trace in the definition of $R_{q}$ (see (2)) has to be taken over the entire matrix $N$.

On the contrary, $R_{v}$ does not show this symmetry. An applied voltage distinguishes the two reservoirs from one another. Thus, the trace in $R_{v}$ (see (2)) cannot be expressed by dwell times only. To clarify the origin of $R_{v}$ we apply a basis transformation in each reservoir and divide the scattering matrix in $2 \times 2$ blocks of the form

$$
s^{(n)}=\left(\begin{array}{cc}
-i \sqrt{R_{n}} e^{i\left(\phi_{n}+\phi_{A, n}\right)} & \sqrt{T_{n}} e^{i\left(\phi_{n}-\phi_{B, n}\right)} \\
\sqrt{T_{n}} e^{i\left(\phi_{n}+\phi_{B, n}\right)} & -i \sqrt{R_{n}} e^{i\left(\phi_{n}-\phi_{A, n}\right)}
\end{array}\right) .
$$

Each block is defined by its transmission probability $T_{n}=$ 
$1-R_{n}$ and three scattering phases $\phi_{n}, \phi_{A, n}, \phi_{B, n}$. Using the definition of $R_{v}$ (Eq. (2) we arrive at [16]

$$
R_{v}=\frac{\sum_{n}\left(\frac{1}{4 T_{n} R_{n}}\left(\frac{d T_{n}}{d E}\right)^{2}+T_{n} R_{n}\left(\frac{d \phi_{A, n}}{d E}+\frac{d \phi_{B, n}}{d E}\right)^{2}\right)}{\left(\sum_{n} \frac{d \phi_{n}}{d E}\right)^{2}} .
$$

Eq. (7) can be connected to earlier results [1, 6, 7] in the infinite capacitance limit where $C_{\mu}^{2} R_{v}$ in Eq. (4) can be replaced by $D_{\mu}^{2} R_{v}$. Eq. (7) has an interesting physical interpretation that gets a particularly appealing form (7) in the scattering formalism used here: The energy derivatives $d T_{n} / d E$ express the sensitivity of the conductance to a potential variation $\Delta U$ on the $\mathrm{MC}$ $(d / d E=-\partial / \partial(e U))$. If this sensitivity is high, the MC is a fast measuring device to determine the position of the electron on the $\mathrm{DD}$. We get a large $R_{v}$ and therefore a fast decoherence rate due to the applied voltage $|V|$. The decoherence rate is thus coupled to the speed of the measurement process.

The measurement is described by a measurement time [6.14] $\tau_{m}=4 S_{I I} /(\Delta I)^{2}$ which is needed for a signal to noise ratio of 1 . Here $S_{I I}$ denotes the low frequency shot noise spectrum and $\Delta I=I_{1}-I_{2}$ is the difference of current flowing through the $\mathrm{MC}$ depending on the state of the two-level system. This difference is evaluated by use of the Landauer formula

$$
\Delta I=\Delta G|V|=\frac{e^{2}}{2 \pi}|V| \sum \frac{d T_{n}}{d E}(e \Delta U)
$$

where $\Delta G$ is the change of conductance between the two states of the double dot and $\Delta U=e C_{\mu} / D\left(C_{i}-C_{\mu}\right)$ the potential change on the MC. The shot noise is as usual $S_{I I}=e|V|\left(e^{2} / 2 \pi\right) \sum R_{n} T_{n}$. Using weak coupling $C_{1}, C_{2} \ll C_{i}$ one gets for the inverse measurement time

$$
\tau_{m}^{-1}=\Gamma_{m}=2 \pi\left(\frac{C_{\mu}}{C_{i}}\right)^{2} R_{m} e|V|
$$

with the dimensionless constant

$$
R_{m}=\frac{1}{4} \frac{\left(\sum \frac{d T_{n}}{d E}\right)^{2}}{\left(\sum \frac{d \phi_{n}}{d E}\right)^{2}\left(\sum R_{n} T_{n}\right)} .
$$

It is not difficult to show that $R_{m} \leq R_{v}$ [19] which leads to an important inequality between measurement rate and decoherence rate $\Gamma_{m} \leq \Gamma_{d e c}$. The measurement is always slower than the decoherence, the decay of the offdiagonal elements of the reduced density matrix of the two-level system.

Which conditions are needed to get the equality $\tau_{m}=$ $\Gamma_{d e c}^{-1}$ ? The tunneling between the two double dots during the measurement must be negligible, $\Delta \simeq 0$, and the temperature must be much smaller than the applied voltage $k T \ll e|V|$. More interesting are three constraints imposed by the scattering matrix (let the $\mathrm{MC}$ be defined by an equilibrium electrostatic potential $\mathrm{V}(\mathrm{x}, \mathrm{y}, \mathrm{z})$; let the scattering states be extended in the z-direction and confined in the xy-plane):

- In order to have $d \phi_{B, n} / d E=0$ in Eq. (7) the scattering Hamiltonian must obey time-reversal symmetry.

- Furthermore, the derivatives $d \phi_{A, n} / d E$ have to vanish. This can be the case accidentally but is always fulfilled for symmetric detectors that obey an inversion symmetry $V(x, y, z)=V(x, y,-z)$. This condition is well known, see for instance [14]. A quantum-limited measurement can only be reached by a spacially symmetric detector. Otherwise part of the information about the state of the DD is transferred to the phase of the scattered electrons. This phase does not influence a conductance measurement.

- In the multichannel case $N>1$ another condition is needed! The equality $R_{m}=R_{v}$ then implies that

$$
\frac{d T_{n} / d E}{R_{n} T_{n}}=C(E) .
$$

The function $C(E)>0$ does not depend on the index $n$ ! This restriction is of statistical origin: The total conductance of the detector is a sum of one channel conductances that have independent uncertainties. Under condition (11) the statistical uncertainty of their sum is minimized. Eq. (11) can be interpreted as differential equations for the transmission probabilities $T_{n}$. Their solutions are all of the form $T_{n}=\left(1+e^{-\left(F(E)-F\left(E_{n}\right)\right)}\right)^{-1}$ with $d F / d E=C$ (The function $F$ is therefore monotonously increasing). The only difference allowed between the different probabilities $T_{n}$ is the offset energy $E_{n}$.

The simplest case is that of a tunnel contact: In this case the probabilities $T_{n}$ are dominated by the action in the forbidden region and Eqs. (11) are independent of the channel number. Thus a tunnel barrier is an efficient detector but has the drawback that its measurement time is long.

Detectors with shorter measurement times can be achieved in structures with higher transparencies. For such structures the condition Eq. (11) is now important. Transmission probabilities of the type (11) occur automatically if the scattering problem is separable due to a potential of shape

$$
\mathrm{V}(\mathrm{x}, \mathrm{y}, \mathrm{z})=\mathrm{Z}(\mathrm{z})+\mathrm{W}(\mathrm{x}, \mathrm{y}) .
$$

This occurs e.g. for the case $F=2 \pi E / \omega_{z}$ with a symmetric harmonic scattering potential $Z(z)=V_{0}-m \omega_{z}^{2} z^{2} / 2$.

We demonstrate the importance of our findings with a generic model that violates condition (12). This condition states that a geometry with a separable potential $V(x, y, z)=Z(z)+Y(x, y)$ is favorable to obtain an efficient detector in the case of more than one open channel. It is clear that a chaotic potential violates condition (12) by definition. We expect therefore that chaos reduces drastically the efficiency $\Gamma_{m} / \Gamma_{v}=R_{m} / R_{v}$ of a detector with two open channels, but has little impact on a detector with only one open channel. 


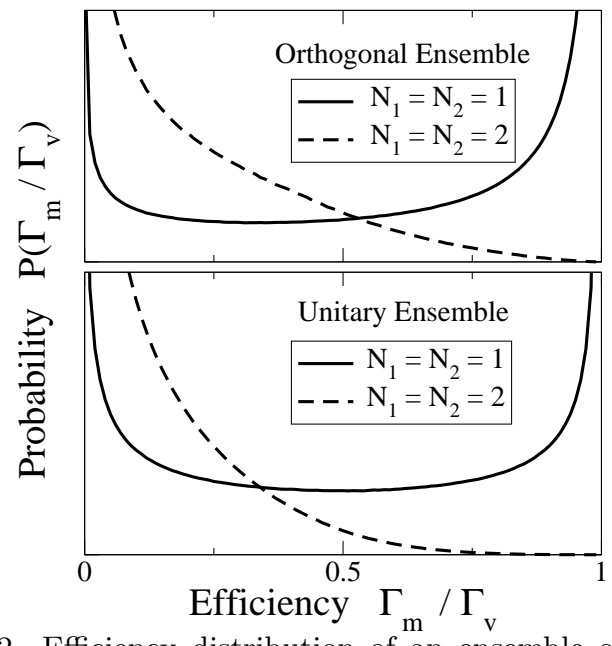

FIG. 2. Efficiency distribution of an ensemble of chaotic quantum cavity detectors: orthogonal ensemble (top panel), unitary ensemble (lower panel) for single channel $\left(N_{1}=N_{2}=1\right)$ and double channel $\left(N_{1}=N_{2}=2\right)$ point contacts.

To check this expectation we use a common model of a chaotic cavity coupled to a left lead with $N_{1}$ channels and a right lead with $N_{2}$ channels: it can be described by a scattering matrix belonging to the circular ensemble of random matrix theory [20]. The distribution of the density of states matrix elements (11) is also known [21. Using these distributions it is straight forward to obtain the probability distribution of the measurement efficiency $R_{m} / R_{v}$

$$
\begin{gathered}
P\left(R_{m} / R_{v}\right)= \\
\int d s \int d N_{E} \delta\left(R_{m} / R_{v}-R_{m}\left(s, N_{E}\right) / R_{v}\left(s, N_{E}\right)\right) .
\end{gathered}
$$

In Eq. (13) $d s$ is a measure for the circular ensemble of scattering matrices and $d N_{E}$ a measure for the symmetrized density of states matrix $N_{E}=$ $s^{-1 / 2} d s / d E s^{-1 / 2} / 2 \pi i$. It turns out that the ratio $R_{m} / R_{v}$ depends only on the eigenvectors of $N_{E}$ and the scattering matrix, but not on the eigenvalues of $N_{E}$, the inverse dwell times $\tau_{n}^{-1}$. The distribution of $R_{m} / R_{v}$ is therefore the same in the canonical $(C \ll D)$ and grand-canonical ensemble $(C \gg D)$. Their difference is explained in [22. Fig. 2 shows the distribution of the measurement efficiency $R_{m} / R_{v}$ in the orthogonal (time-reversal symmetry) and unitary ensemble (broken time-reversal symmetry). The distributions were obtained by numerical integration. The distribution for $N_{1}=N_{2}=1$ in the unitary ensemble can also be calculated analytically to be $P\left(R_{m} / R_{v}\right)=\left(R_{m} / R_{v}\left(1-R_{m} / R_{v}\right)\right)^{-1 / 2}$. Surprisingly, despite the absence of inversion symmetry a chaotic dot with open single channel contacts is with high probability an efficient detector! It is clearly visible that chaos reduces strongly the efficiency of the measurement device as soon as more than one channel contributes to the electric transport. The reduction due to a broken timereversal symmetry is much less pronounced.
In this work we have analyzed coherent multichannel mesoscopic conductors with the aim to find both fast and efficient detectors. We find a new statistical condition necessary to carry out a quantum-limited measurement. This condition relates sensitivities and shot noises of different conductance channels. It leads us to a class of detectors (defined by separable potentials) that are both fast and efficient. We have assumed that a change in the state of the system causes only a small change in the potential landscape of the detector. Only under this condition is it possible to describe the detector response with the help of small differential changes of the scattering matrix and linear screening. We leave it as a future challenging problem to develop a theory of phase-coherent, non-linear mesoscopic detectors.

This work was supported by the Swiss National Science Foundation.

[1] E. Buks, R. Schuster, M. Heiblum, D. Mahalu, V. Umansky, and H. Shtrikman, Nature 385, 417 (1997).

[2] D. Sprinzak, E. Buks, M. Heiblum, and H. Shtrikman, Phys. Rev. Lett. 84, 5820 (2000).

[3] M. Field, et al., Phys. Rev. Lett. 70, 1311 (1993).

[4] R. A. Harris and L. Stodolsky, Phys. Lett. B 116, 464 (1982).

[5] M. Büttiker and A. M. Martin, Phys. Rev. B 61, 2737 (2000).

[6] I. L. Aleiner, N. S. Wingreen, and Y. Meir, Phys. Rev. Lett. 79, 3740 (1997).

[7] Y. Levinson, Europhys. Lett. 39, 299 (1997).

[8] A. Silva and S. Levit, Phys. Rev. B 63, 201309(R) (2001).

[9] S. A. Gurvitz, Phys. Rev. B 56, 15215 (1997).

[10] Y. Makhlin, G. Schön, and A. Schnirman, Rev. Mod. Phys. 73, 357 (2001).

[11] A. N. Korotkov, Phys. Rev. B 63, 115403 (2001).

[12] H. S. Goan and G. J. Milburn, Phys. Rev. B 64, 235307 (2001).

[13] D. V. Averin, cond-mat/0010052 (2000).

[14] A. N. Korotkov and D. Averin, Phys. Rev. B 64, 165310 (2001).

[15] H. J. Carmichael, Statistical Methods in Quantum Optics 1, Master Equations and Fokker-Planck Equations, ISBN 3-540-54882-3, Springer, Berlin 1999.

[16] M. H. Pedersen, S. A. van Langen, and M. Büttiker, Phys. Rev. B 57, 1838 (1998).

[17] M. Büttiker, H. Thomas, and A. Prêtre, Phys. Lett. A 180, 364 (1993).

[18] P. W. Brouwer, and M. Büttiker, Europhys. Lett. 37, 441 (1997).

[19] The relation $R_{m} \leq R_{v}$ has the form of a Cauchy-Schwarz inequality.

[20] C. W. J. Beenakker, Rev. Mod. Phys. 69, 731 (1997).

[21] P. W. Brouwer, K. M. Frahm, and C. W. J. Beenakker, Phys. Rev. Lett. 78, 4737 (1997).

[22] P. W. Brouwer, et al., Phys. Rev. Lett 79, 913 (1997). 